Results Following application of statistical (machine) learning methods described, a 17 gene qPCR assay was developed and optimised. The validation cohort of 123 patients, could be classified into two distinct subgroups, IBD ${ }^{\text {hi }}$ (high risk) and $\mathrm{IBD}^{\mathrm{lo}}$ (lower risk). Irrespective of the underlying diagnosis, IBD $^{\text {hi }}$ patients experienced significantly more aggressive disease than $\mathrm{IBD}^{\mathrm{lo}}$ patients, with an earlier need for treatment escalation (hazard ratio=2.65 (CD), $3.12(\mathrm{UC})$ ).

Subsequently, this biomarker is being used to stratify therapy in the PROFILE trial, where 45 sites have been opened and at the time of writing, over 260 participants randomised - with recruitment ongoing.

Conclusions We have developed, optimised and validated a whole-blood qPCR classifier that is able to predict disease course from diagnosis in patients with IBD. This classifier is currently being used in the PROFILE trial, the first biomarker-stratified trial in Gastroenterology, and if the clinical utility of a stratified treatment approach is demonstrated this would represent a major step towards personalised therapy in IBD.

\section{IDDF2020-ABS-0204 FECAL MICROBIOTA AS A NON-INVASIVE IDENTIFICATION FOR POSTOPERATIVE ENDOSCOPIC RECURRENCE IN CROHN'S DISEASE PATIENTS}

${ }^{1}$ Haichao Wang*, ${ }^{2}$ Guorong Yan, ${ }^{1}$ Yaling Wu, ${ }^{3}$ Chen Ye, 'Deji Zhuoma, ${ }^{1}$ Pengyu Yang, ${ }^{1}$ Lei Zhang, ${ }^{4}$ Chungiu Chen, 'Xiaolei Wang. ${ }^{1}$ Department of Gastroenterology, Shanghai Tenth People's Hospital, Tongji University School of Medicine, China; ${ }^{2}$ Institute of Photomedicine, Shanghai Skin Disease Hospital, Tongji University School of Medicine, China; ${ }^{3}$ Medical College of Soochow University, China; ${ }^{4}$ Center for Difficult and Complicated Abdominal Surgery, Shanghai Tenth People's Hospital, Tongji University School of Medicine, China

\subsection{6/gutjnl-2020-IDDF.10}

Background Postoperative recurrence (POR) is extremely frequent in Crohn's disease (CD) after ileocolonic resection and usually assessed by endoscopy. However, the use of endoscopies is limited by its invasiveness. Dysbiosis of gut microbiota has been implicated in triggering POR. Here, we aimed to establish a non-invasive tool based on fecal microbiota to identify POR in Chinese CD patients.

Methods Fecal samples from 52 CD patients within 12 months after ileocecal resection were collected for gut microbiota analysis using 16S rRNA genes sequencing technology. POR was defined by a Rutgeerts score $\geq \mathrm{i} 2$ under endoscopy at the time of sample collection. Microbiota abundance at the genus level was employed to construct the model to estimate POR by logistic regression model (LRM). Leave-one-out cross validation was used to evaluate the robustness of the model. Receiver operating characteristic curve analysis was performed to test the sensitivity and specificity of the microbial model.

Results Significant reduction of microbial diversity was noted in the POR group $(p<0.05)$. In patients developing POR (27 cases), an increase of Flavonifactor.spp belonging to Ruminococcaceae family, and a reduction of 14 genera, including three short-chain fatty acid (SCFA)-producing genera belonging to Lachnospiraceae family (Stomatobaculum. spp, Oribacterium.spp and Eubacterium.spp), was observed (all $p<0.05$, (figure 1A)). Lower percentage of infliximab treatment was found in POR patients $(33.3 \%$ vs. $61.5 \%$, $p$
A

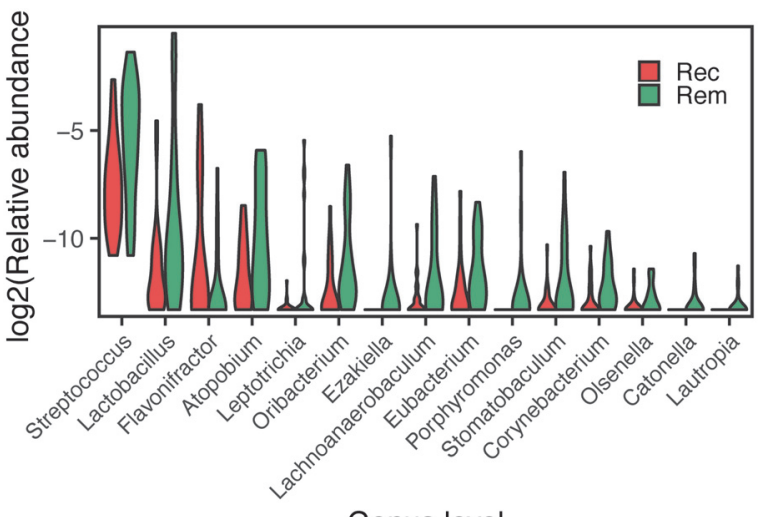

Genus level

Abstract IDDF2020-ABS-0204 Figure 1A Identification of discriminatory gut microbiota for $C D$ patients with postoperative recurrence

\section{B}

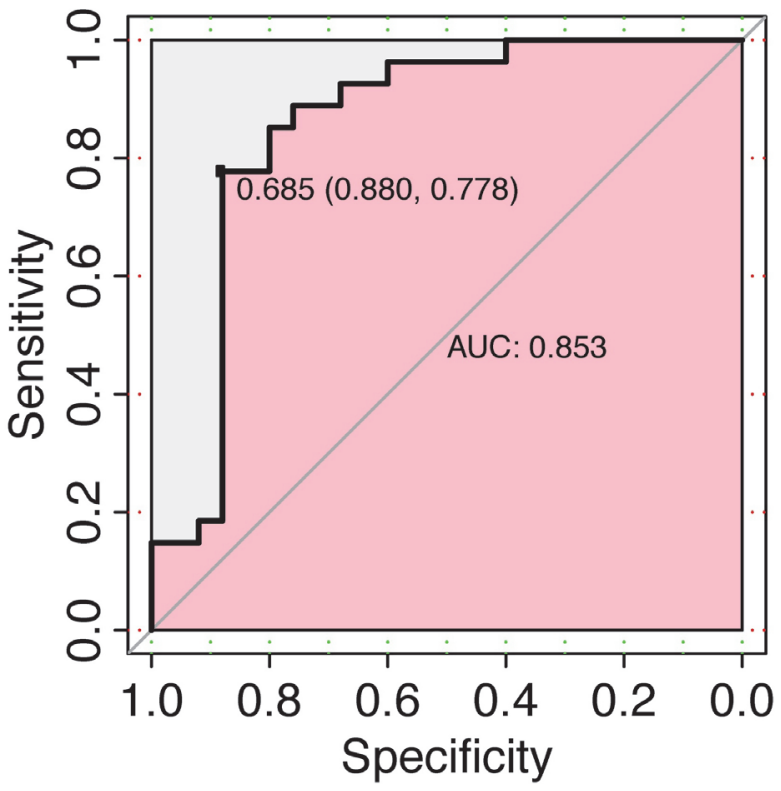

Abstract IDDF2020-ABS-0204 Figure 1B Identification of discriminatory gut microbiota for $C D$ patients with postoperative recurrence

$=0.040$ ), and closely associated with elevated abundance of Stomatobaculum.spp and Oribacterium.spp $(p<0.01)$. Both the abundance of Stomatobaculum.spp and Oribacterium.spp were negatively correlated with the counts of platelets or lymphocytes $(p<0.05)$. A score combined with discriminatory microbial species was calculated to predict POR, and achieved an area under curve value of 0.853 (0.880 for specificity, 0.778 for sensitivity, (Figure 1B)). The correlation coefficient between the observed and the predicted values was $0.651(p<0.01)$.

Conclusions POR is tightly linked to the changes in microbiota composition and diversity. Fecal microbiota, including Flavonifactor.spp and three genera of SCFA-producing Lachnospiraceae family, represents a non-invasive biomarker for diagnosis of POR, helping to prevent POR in CD patients. 